\title{
O bem-estar de pacientes renais crônicos durante o tratamento com hemodiálise e diálise peritoneal
}

The well-being of chronic renal patients during treatment with hemodialysis and peritoneal dialysis

El bienestar de los pacientes renales crónicos durante el tratamiento con hemodiálisis y diálisis peritoneal

Maria Adriana Mota Rocha ${ }^{1 *}$, Rosinete Souza Barata², Letícia Cardoso Braz³.

\section{RESUMO}

Objetivo: Avaliar qual método dialítico traz mais qualidade de vida ao paciente com insuficiência renal crônica. Métodos: Foi realizada revisão de literatura do tipo narrativa de artigos dos sites SciELO e uma monografia. Resultados: Os métodos dialíticos, diálise peritoneal e hemodiálise são semelhantes quando se trata de qualidade vida, pois ambos apresentam vantagens e desvantagens ao paciente. Conclusão: $A$ escolha do método dialítico desempenha papel importante para a qualidade de vida do paciente renal crônico, fatores individuais precisam ser analisados e deve ser uma escolha conjunta entre médico família e paciente, visando uma promoção maior da qualidade de vida deste.

Palavras-chave: Qualidade de Vida, Diálise Peritoneal, Hemodiálise.

\begin{abstract}
Objective: To evaluate which dialysis method brings more quality of life to patients with chronic renal failure. Methods: A literature review of the narrative type of articles from SciELO sites and a monograph was carried out. Results: Dialysis methods, peritoneal dialysis and hemodialysis are similar when it comes to quality of life, since both have advantages and disadvantages to the patient. Conclusion: The choice of dialysis method plays an important role in the quality of life of the chronic renal patient, individual factors need to be analyzed and should be a joint choice between family doctor and patient, aiming at a higher quality of life.
\end{abstract}

Keywords: Quality of Life, Peritoneal dialysis, Hemodialysis.

\section{RESUMEN}

Objetivo: Evaluar qué método dialítico trae más calidad de vida al paciente con insuficiencia renal crónica. Métodos: Se realizó una revisión de literatura del tipo narrativa de artículos de los sitios SciELO y una monografía. Resultados: Los métodos dialíticos, diálisis peritoneal y hemodiálisis son similares cuando se trata de calidad de vida, pues ambos presentan ventajas y desventajas al paciente. Conclusión: La elección del método dialítico desempeña un papel importante para la calidad de vida del paciente renal crónico, factores individuales necesitan ser analizados y debe ser una elección conjunta entre médico familiar y paciente, buscando una promoción mayor de la calidad de vida de éste.

Palabras clave: Calidad de vida, Diálisis peritoneal, La hemodiálisis.

\footnotetext{
${ }^{1}$ Enfermeira Nefrologista e do Trabalho- Universidade Federal da Bahia, Salvador-BA.

*E-mail: adrianamotarocha@hotmail.com

${ }^{2}$ Enfermeira Especialista em Enf. Médico-Cirúrgica sob forma de residência - Área de Concentração UTI, pela Universidade Federal da Bahia, Salvador-BA.

${ }^{3}$ Enfermeira, Mestre em Enfermagem pela Universidade Estadual de Feira de Santana, Salvador-BA.
}

SUBMETIDO EM: 10/2018

ACEITO EM: 11/2018

PUBLICADO EM: $3 / 2019$

REAS/EJCH | Vol. Sup. 21 | e670 | DOI: https://doi.org/10.25248/reas.e670.2019 Página 1 de 7 


\section{INTRODUÇÃO}

A doença renal é considerada um grande problema de saúde pública, porque causa elevadas taxas de morbidade e mortalidade e, além disso, tem impacto negativo sobre a Qualidade de Vida Relacionada à Saúde (QVRS). A QRVS é a percepção da pessoa de sua saúde por meio de uma avaliação subjetiva de seus sintomas, satisfação e adesão ao tratamento.

Em 2000 no Brasil 42 mil pessoas sofriam de Insuficiência renal crônica, em 2016 esse número passou para 122 mil pessoas números da Sociedade Brasileira de Nefrologia (SBN, 2017). Ainda segundo a Sociedade Brasileira de Nefrologia ano passado 5,7 mil pessoas realizaram transplante renal, a cada ano 0 número de transplante aumenta $10 \%$.

A insuficiência renal crônica é uma doença que pode resultar tanto da genética como do estilo de vida do portador da doença, entre suas maiores complicações está a falência múltipla dos rins, impedindo que exerça sua principal função a filtragem do sangue, de forma a acarretar um progressivo acúmulo de catabólicos no sangue (THOMASL TV e ALCHIERILL JC; 2005).

A Sociedade Brasileira de Nefrologia preconiza que as pessoas optem pela prevenção, hábitos alimentares saudáveis, fatores genéticos devem ser considerados e o hábito de consumir água regulamente, além da prática de exercícios físicos. Infelizmente depois de descoberta a doença o paciente possui limitações, como consumo de alimentos com quantidade excessiva de líquidos já que os rins não conseguem mais filtrar o sangue como deveria, assim o acúmulo dos catabólicos geram inchaço, uma produção insuficiente de urina onde os mesmos são eliminados (SBN, 2017).

A Insuficiência Renal Crônica já se mostra não possuir cura, pois todas as doenças crônicas são incuráveis. O tratamento mais comum até que se consiga um transplante é o dialítico contínuo (SBN, 2012).

A diálise visa repor as funções dos rins sendo um tratamento invasivo, mas que aumenta a expectativa de vida do paciente, as substâncias tóxicas são retidas, juntamente com a água e sais minerais que se encontram em excesso, estabelecendo assim uma nova situação de equilíbrio (THOMASL TV e ALCHIERILL JC; 2005).

O objetivo do presente trabalho é analisar o atual tratamento de diálise, abordando os benefícios e as alterações que o tratamento de diálise peritoneal e hemodiálise provocam na qualidade de vida do paciente.

\section{MÉTODOS}

Trata-se de uma revisão de literatura do tipo narrativa, que segundo, Souza MT et al. (2010), é um método que proporciona a síntese de conhecimento e a incorporação da aplicabilidade de resultados de estudos significativos na prática.

As bases de dados utilizadas foram a Scientific Eletronic Library Online (SCielo) e Analysis and Retrial Sistem On-line (Medline). Foram incluídos artigos de 2005 a 2017.

Os procedimentos de coleta de dados para a realização da pesquisa foram através das palavras-chave: Qualidade de vida, Diálise peritoneal, Hemodiálise.

Após passar pelos critérios de inclusão que foram artigos científicos, estudos clínicos, revisões sistemáticas da literatura e relatos de caso, que abordaram o tema proposto, publicados entre os anos de 2005 e 2017, na língua inglesa e portuguesa.

Quanto aos critérios de exclusão, não foram escolhidos estudos publicados fora dos anos de investigação, que não abordaram o tema proposto, restando 50 artigos aos quais foram lidos os títulos e selecionados os que condiziam com o tema do trabalho, restando 25 artigos.

Após essa seleção foram lidos os resumos de forma minuciosa para identificar os que atendiam ao objetivo da pesquisa, de forma que restaram 6 artigos. 


\section{RESULTADOS}

Tabela 1 - Composição dos artigos utilizados.

\begin{tabular}{|c|c|c|c|c|}
\hline AUTOR & TÍTULO & PERIÓDICO & ANO & OBJETIVO \\
\hline ARENAS VG & $\begin{array}{l}\text { Qualidade de Vida: } \\
\text { comparação entre } \\
\text { diálise peritoneal } \\
\text { automatizada e } \\
\text { hemodiálise. }\end{array}$ & Acta Paul Enferm. & 2009 & $\begin{array}{c}\text { Avaliar a qualidade de } \\
\text { vida relacionada à saúde } \\
\text { em pacientes submetidos } \\
\text { à Diálise Peritoneal } \\
\text { Automatizada e } \\
\text { Hemodiálise em um } \\
\text { centro de diálise satélite } \\
\text { no Município de São } \\
\text { Paulo. }\end{array}$ \\
\hline $\begin{array}{l}\text { GONÇALVES } \\
\text { FA }\end{array}$ & $\begin{array}{c}\text { Qualidade de vida } \\
\text { de pacientes renais } \\
\text { crônicos em } \\
\text { hemodiálise ou } \\
\text { diálise peritoneal: } \\
\text { estudo comparativo } \\
\text { em um serviço de } \\
\text { referência de } \\
\text { Curitiba - PR }\end{array}$ & $\begin{array}{l}\text { Brazilian Journal de } \\
\text { Nephrology.Jornal } \\
\text { Brasileiro de } \\
\text { Nefrologia. }\end{array}$ & 2015 & $\begin{array}{l}\text { Comparar a qualidade de } \\
\text { vida de pacientes renais } \\
\text { crônicos em estágio V-D } \\
\text { (em diálise) que realizam } \\
\text { DP domiciliar ou HD. }\end{array}$ \\
\hline
\end{tabular}

\begin{tabular}{cccc}
\hline SILVA GE & $\begin{array}{c}\text { Qualidade de vida } \\
\text { do paciente renal } \\
\text { crônico em }\end{array}$ & $\begin{array}{c}\text { PEPSIC- Periódicos } \\
\text { Eletrônicos em }\end{array}$ & 2011 \\
tratamento & Psicologia & $\begin{array}{c}\text { Identificar a qualidade de } \\
\text { vida de pacientes renais } \\
\text { crônicos em hemodiálise }\end{array}$ \\
& & $\begin{array}{c}\text { numa Clínica do Rim em } \\
\text { Dourados. }\end{array}$
\end{tabular}

\begin{tabular}{lcccc}
\hline $\begin{array}{l}\text { MACHADO } \\
\text { GRG }\end{array}$ & $\begin{array}{c}\text { Tratamento de } \\
\text { diálise em pacientes } \\
\text { com insuficiência } \\
\text { renal crônica. }\end{array}$ & Cadernos UniFOA & 2014 & $\begin{array}{c}\text { Abordar os benefícios e } \\
\text { as alterações que o } \\
\text { tratamento de diálise } \\
\text { provoca na qualidade de } \\
\text { vida do paciente. }\end{array}$ \\
\hline $\begin{array}{l}\text { RAMALHO } \\
\text { NETO JM }\end{array}$ & $\begin{array}{c}\text { Fístula } \\
\text { Arteriovenosa na } \\
\text { perspectiva de } \\
\text { pacientes. }\end{array}$ & $\begin{array}{c}\text { Enfermagem em } \\
\text { Foco. }\end{array}$ & 2016 & $\begin{array}{c}\text { Analisar a percepção de } \\
\text { pacientes renais crônicos } \\
\text { frente à fítula }\end{array}$ \\
& & & $\begin{array}{c}\text { arteriovenosa implantada } \\
\text { em seu corpo. }\end{array}$ \\
\hline
\end{tabular}

\begin{tabular}{ccccc}
\hline MARINHO CLA & $\begin{array}{c}\text { Qualidade de vida } \\
\text { de pessoas com } \\
\text { doença renal crônica } \\
\text { em hemodiálise }\end{array}$ & $\begin{array}{c}\text { Rev. Rene-Revista } \\
\text { da Rede de } \\
\text { Enfermagem do } \\
\text { Nordeste }\end{array}$ & $\begin{array}{c}\text { Analisar a qualidade de } \\
\text { vida de pessoas com } \\
\text { doença renal crônica. }\end{array}$ \\
& & ach
\end{tabular}

Fonte: Próprias autoras, 2017. 


\section{DISCUSSÃO}

A insuficiência renal crônica é uma doença que pode resultar tanto da genética como do estilo de vida do portador da doença, entre suas maiores complicações está a falência múltipla dos rins, impedindo que exerça sua principal função a filtragem do sangue, de forma a acarretar um progressivo acúmulo de catabólicos no sangue (MACHADO e PINHATI; 2014).

Machado; Pinhati (2014) explicam que a IRC é um diagnóstico de uma síndrome que evidencia a perda progressiva e, geralmente irreversíveis provocadas por doenças que tornam o rim incapaz de realizar suas funções. Com uma evolução lenta, mas de forma progressiva. As perdas são de forma gradativa, assim tornando o organismo adaptado a cada mudança, o que dificulta o diagnóstico, já que mantêm o paciente sem sintomas da doença. Os medicamentos e uma dieta restritiva, são recomendadas quando o rim está apenas com $12 \%$ de sua capacidade funcional.

Para a SBN (2012), o rim com capacidade de funcionamento de $12 \%$, já está em um estado crítico para os pacientes, por isso a adoção de métodos com tratamento medicamentosos, ou com a utilização das máquinas que oferecem a filtragem do sangue, função que deveria ser exercida pelos rins são exemplos, diálise ou transplante renal, em 2016 foram realizados 5.492 transplantes de rins. IRC é um grave problema de saúde pública no mundo todo, uma epidemia de crescimento assustador. O diagnóstico ainda é um pouco complicado, por diversos fatores como, a falta de informação sobre a doença, a demora na realização dos exames, e o acesso dificultado às unidades de saúde básica no país. Com o diagnóstico preciso o paciente deve ser submetido a um tratamento conservador ou dialítico o mais precoce possível, pois as ocorrências de complicações podem levar à morte. Uma dieta balanceada, um acompanhamento com médicos, nutricionistas, em alguns casos farmacêuticos, são indispensáveis para manutenção da qualidade de vida dessas pessoas.

Arena et al. (2009) esclarece que o programa dialítico é inserido quando o tratamento mais comum, somente com a dialise não é capaz de manter a qualidade de vida do paciente, é importante colocar para esses pacientes que os mesmos podem ter uma vida tranquila, seguindo as recomendações de seus médicos, e profissionais da saúde que os acompanham e assim quando ocorre o surgimento de sintomas importantes da uremia, podendo ser realizado através de diálise peritoneal ou a hemodiálise. Os cuidados ao paciente com IR devem acontecer de forma humanizada, passando respeito, dignidade, para esse paciente se sentir acolhido e bem cuidado pelo profissional de saúde.

Madeiro et al. (2010); Machado; Pinhati (2014) concordam que pensando em uma melhor a eficácia da hemodiálise é coerente que os pacientes assumam o tratamento dialítico como alternativa na recuperação da sua qualidade de vida. A hemodiálise é um procedimento de filtração de resíduos extra corporais do sangue realizado pela máquina dialisador, assim substituindo as funções renais. Assim para começar o tratamento, uma fístula é inserida cirurgicamente arteriovenosa ou um cateter específico na veia.

Ramalho Neto et al. (2016) durante a hemodiálise, uma parte do sangue do paciente passa pela fístula ou cateter específico, onde chega até o dialisador, pela linha lateral, onde ocorre a filtração, retornando ao paciente pela linha venosa. O paciente necessita de três a quatro sessões de hemodiálise por semana, a duração das sessões pode variar de até 4 horas de duração. A qualidade de vida do paciente também é importante para realização da sessão, se o mesmo possui uma vida estável, sem maiores complicações por causa da doença, as sessões ocorrem sem interrupções. Não há como diminuir a importância da contribuição que os avanços tecnológicos trouxeram ao nosso cotidiano, e baseando-se nesse avanço devemos cada vez mais dar importância à dimensão humana, psicológica e cultural da doença.

Em estudo realizado por Gonçalves et al. (2015) foi possível entender que na realização das sessões, se o paciente se encontra em estado crítico, pelo seu estilo de vida, não responde bem aos medicamentos, se alimenta de forma errônea, a hemodiálise não é executada da forma correta, os efeitos colaterais são bem comuns para os pacientes, mas se as recomendações como a ingestão mínima de sais minerais e água, além de alimentos naturais e que não sejam a base de sódio forem evitados, esses efeitos podem diminuir bastante. Os pacientes relatam que as cãibras musculares e a hipotensão são muito comuns, ficando fraco e com 
tontura além das náuseas. O tratamento medicamentoso diminui esses efeitos, por isso a importância de seguir à risca os horários, a dieta também é de extrema importante. A hemodiálise é a substituição das funções renais, beneficiando o paciente para ter uma melhor qualidade de vida, assim é possível o monitoramento dos níveis plasmáticos de potássio, ureia, sódio e cloretos.

Já Ramos (2014) diz que a dialise peritoneal (DP) tem o mesmo objetivo da hemodiálise e pode substituir no tratamento dando os mesmos efeitos para o paciente que sofre de IR. Esse processo funciona com o peritônio, uma membrana que fica no abdome, ela que age como o dialisador, assim como é semipermeável, filtra o sangue de maneira eficaz. O conhecimento profissional constitui no exercício do conhecimento, aplicado a prática e visando à promoção da saúde, em busca de proporcionar sempre o melhor de si no atendimento para melhorar a qualidade de vida do paciente.

Gonçalves et al. (2015) informa que a dialise peritoneal utiliza uma solução de dialise, o que é bem conhecida como banho de dialise, isso para perfurar na realização da manobra. Com o auxílio de uma bolsa que passa a dialise até o cateter na cavidade abdominal, isso demora de tal forma, que a bolsa permanece lá durante horas. Assim se drena essa solução, depois uma nova solução é colocada e volta preenchendo o abdômen por completo, e essa manobra renova toda a manobra de depuração. Dependendo do estado de saúde do paciente, as trocas vão ocorrer de três á seis vezes ao dia. Os profissionais de saúde ao realizar as manobras de trocas devem estar atentos para que não ocorram eventuais problemas como infecções. As crianças e adolescentes quando sofrem de IR os seus médicos optam por esse tratamento, por ser menos invasivo. Diversas vantagens podem ser oferecidas pela escola da DP, desde um combate da uremia bioquímico, até mesmo da anemia e hipertensão, algumas funções podem ser preservadas como a renal residual, de forma que o paciente possa se alimentar menos restritamente, e ingerir uma quantidade maior de líquidos, para as crianças e os adolescentes isso é muito importante, já que restringir alguns alimentos nessa fase da vida humana, é muito mais complicado. Os riscos existem como em qualquer tratamento, mas isso somente se o paciente não seguir as recomendações, como a infecção do peritônio conhecida como peritonite, a família muitas vezes desiste do tratamento, quando há esse tipo de ocorrência, um tratamento que consiste de toda uma vida, possui seus momentos de maior gravidade, até que enfim esse paciente consiga ser transplantado, sua vida não volta a rotina normal por completo, mas a diminuição de idas ao hospital, já é um alivio para muitos. Com início no orifício de saída cutâneo a infecção pode causar a morte do paciente, assim o cateter não pode mais permanecer no mesmo local, sua retirada é imediata.

O autor Silva et al. (2011) informa em seu estudo que a infecção do peritônio, pode ser tratada de forma simples, basta ter todos os cuidados técnicos e clínicos durante o tratamento. Pode ocorrer o entupimento do cateter, pois o líquido possui diversos resíduos, mas pode ser resolvido no hospital onde o paciente realiza o procedimento.

Ramos (2014) afirma que os três tipos de diálise peritoneal existentes são compatíveis para exercer de forma satisfatória suas funções, sendo elas: diálise peritoneal ambulatorial contínua (DPAC), a diálise peritoneal cíclica contínua (DPCC) e diálise peritoneal intermitente (DPI).

Em Ramos (2014) é estabelecido que durante todo o processo a solução de diálise fica no abdômen durante sete dias da semana, assim não são necessárias às trocas durante o dia, é bem comum esse tipo de procedimento para os pacientes de IR. A indicação para esse processo que a solução permanece uma semana, é para os pacientes que possuem uma hipersensibilidade, indicada pelo PET (do inglês Peritoneal Equilibrium Test), esses pacientes são geralmente os idosos, as crianças.

Saúde Biazi (2012) explica que o próprio paciente pode realizar o método em casa, pois o mesmo independe de equipamento, assim a infusão e a retirada é feita pelo paciente, uma pessoa treinada pode auxiliar o mesmo durante todo o método, o acesso venoso não é necessário. A questão que pode ser um empecilho é a duração da solução, 4 horas, logo a próxima troca deve ocorrer nesse período. Assim durante o dia é realizada de 4 a 5 trocas, e o tempo para realizar a troca é de 40 minutos. Antes de chegar o tempo máximo de troca, o paciente já deve iniciar esse procedimento, para que não ultrapasse as 4 horas permitidas para a solução permanecer no abdômen. 
No estudo realizado pela Saúde Biazi (2012) fica claro que a DPCC, é bastante parecida com a DPAC, a diferença é na conexão do cateter, que permanece ligado á máquina que filtra automaticamente toda a solução, se realiza a noite, pois dessa forma o paciente possui o dia inteiro para realizar suas outras atividades, essa é uma vantagem da DPCC. Infelizmente a DPA tem um custo elevado na sua utilização, por isso no Brasil o seu uso é pouco e restrito, apesar de ter uma resposta rápida com o tratamento para os pacientes.

Já para Silva et al. (2011) a DPA está sendo escolhida por uma maior parte dos pacientes que são acometidos pela IR, pois oferece á eles uma maior segurança, os índices de infecções são bem menores, o tempo destinado para realização da filtragem é pouco, logo sobrando tempo para exercer outras atividades, maior flexibilidade para a prescrição de diálise para atingir adequação, um controle efetivo durante as sessões, assim um registro é possível de acordo com as ocorrências, e pela melhor qualidade de vida do paciente que utiliza esse método dialítico. O terceiro tipo de diálise peritoneal, a DPI, é feito a partir da utilização de uma máquina, seja para infusão quanto no momento de drenagem da solução. Esse instrumento pode ser do mesmo tipo ao empregado na DPAC. A DPI é feita em unidades de saúde especializadas na realização da mesma, na verdade ela é indicada mais para os pacientes que não podem fazer nenhum outro tipo de diálise por algum impedimento, como paciente com alta permeabilidade de membrana e função renal residual significativa. Com diversas complicações que podem ocorrer com esse método, o DPI é realizado no hospital e dura em média um dia inteiro e a troca ocorre de 2 em 2 horas. É importante ressaltar que o abdômen seco durante as diálises deve permanecer assim. Mesmo com diversos tipos de diálise, todos são importantes e os médicos devem sempre estar cientes de qual seu paciente está realizando, mesmo aqueles que não oferecem a necessidade de estar no hospital todos os dias, assim a família, paciente e equipe médica ficam a vontade de escolher o melhor de acordo com o quadro clínico e qualidade de vida desses pacientes.

Silva et al. (2011) em seu estudo esclarece que a qualidade de vida é um conjunto de medidas que oferecem bem-estar, alimentação adequada, atividade física, cuidados médicos adequados, uso de medicamentos quando necessário, sempre prescritos por um médico ou farmacêutico. Assim os aspectos que conferem o modo de vida, contemplando as esferas de bem-estar físico, mental, desempenho no trabalho e participação social. Definir qualidade de vida é subjetivo, mas se reunir as diversas formas de abordar um método de conviver com a doença é possível ter a definição. Os pacientes tenham o direito de receber uma assistência qualificada, e o cuidado ao paciente IR cabendo às instituições de saúde quantificar o número que atenda a demanda e qualifique esses profissionais para prestar a melhor assistência aos pacientes. Quando adoecem os pacientes podem ter diversas dificuldades, e adaptar suas vidas a essa nova realidade pode ser dificultoso, por isso seguir as recomendações da equipe médica para se ter uma qualidade de vida melhor.

Kirchner et al. (2011) descreve que as dificuldades que podem acontecer aos pacientes com IRC vão interferir no seu meio social, cultural, em algumas situações nas suas crenças, por isso o apoio dos familiares, dos profissionais envolvidos com a recuperação e cuidados desse paciente são essenciais. É importante que o paciente receba tanto de sua família, como da equipe multiprofissional um amparo social, que auxilia na recuperação, ou nos momentos de recaída.

\section{CONSIDERAÇÕES FINAIS}

Os doentes renais crônicos têm sua qualidade de vida radicalmente mudada depois do diagnóstico e tratamento. São indivíduos complexos, não somente pelos fatores físicos, como também os emocionais. Os métodos dialíticos hemodiálise e diálise peritoneal afetam a qualidade de vida destes de forma parecida. Haja vista que ambos deixam os pacientes cansados e deprimidos. Faz-se necessário que os profissionais de saúde, bem como os familiares, promovam um ambiente com o mínimo de stress possível, com um cuidar organizado e integral, permitindo assim uma vivência com maior qualidade, independentemente do método dialítico de escolha ou necessidade. 


\section{REFERÊNCIAS}

1. ARENAS, VG et al. Qualidade de Vida: Comparação entre diálise peritoneal e hemodiálise. Acta Paul Enferm, 2009; 14(30):535-539.

2. GONÇALVES, FA et al. Quality of life in chronic renal patients on hemodialysis or peritoneal dialysis: a comparative study in a referral service of Curitiba - PR. Jornal Brasileiro de Nefrologia, 2015; 37(4):374-467.

3. KIRCHNER, RM. et al. Análise do estilo de vida de renais crônicos em hemodiálise. O Mundo da Saúde. 2011; 35(5): 415-421.

4. MACHADO, GRG, PINHATI, FR. Tratamento de diálise em pacientes com insuficiência renal crônica. Cadernos Unifoa, Volta Redonda, 2014; 26(6): 137-148.

5. MADEIRO, AC. et al. Adesão de portadores de insuficiência renal crônica ao tratamento de hemodiálise. Acta Paulista de Enfermagem, 2010; 23(3): 546-551.

6. MARINHO, CLA et al. Qualidade de vida de pessoas com doença renal crônica em hemodiálise. Rev. Rene: Revista da Rede de Enfermagem do Nordeste, 2017, 37 (2): 78-81.

7. RAMALHO NETO, JM et al. Fístula Arteriovenosa na Perspectiva de Pacientes. Enfermagem em Foco, 2016, 7(1):23-28.

8. RAMOS, ECC. Qualidade de vida na Insuficiência Renal Crônica: comparação entre pacientes em hemodiálise e em diálise peritoneal em Pelotas - RS. 2014. 103 f. Dissertação (Mestrado) - Curso de Pós-Graduação em Saúde Pública- Epidemiologia, Universidade Federal de Pelotas, Pelotas.

9. SAÚDE BIAZI (Brasil). Diálise e Hemodiálise. São Paulo, 2012.

10. SILVA, GE et al. Qualidade de vida do paciente renal crônico em tratamento hemodialítico em Dourados. Pepsic, 2011; 15(15): 234-239.

11. SOCIEDADE BRASILEIRA DE NEFROLOGIA (Brasil). Doenças comuns. Tratamento. São Paulo, 2012.

12. SOUZA, MT de, SILVA, MD, CARVALHO, R de. Revisão integrativa: o que é e como fazer. Einstein, Morumbi, 2010; 8(1): 102-106.

13. THOMASL, TV, ALCHIERILL, JC. Qualidade de vida, depressão e características de personalidade em pacientes submetidos à Hemodiálise. Avaliação Psicológica, 2005; 4(2): 57-64. 